\title{
Trois âges d'une migration française au coeur du continent : de la Haute Louisiane espagnole des années 1790 au Missouri de 1860
}

Soazig Villerbu

Volume 73, numéro 1-2, été-automne 2019

L’Ouest franco-américain

URI : https://id.erudit.org/iderudit/1068791ar

DOI : https://doi.org/10.7202/1068791ar

Aller au sommaire du numéro

\section{Éditeur(s)}

Institut d’histoire de l’Amérique française

ISSN

0035-2357 (imprimé)

1492-1383 (numérique)

Découvrir la revue

Citer cet article

Villerbu, S. (2019). Trois âges d'une migration française au coeur du continent : de la Haute Louisiane espagnole des années 1790 au Missouri de 1860. Revue d'histoire de l'Amérique française, 73(1-2), 71-101.

https://doi.org/10.7202/1068791ar

\section{Résumé de l'article}

Les migrations françaises vers l'ancien Pays des Illinois ne s'arrêtent pas avec la fin du Régime français. Dans l'espace qui fut la Haute Louisiane espagnole avant de devenir l'État du Missouri, entre les années 1790 et 1860, cet article définit trois âges de cette migration. Elle est d'abord insérée dans l'histoire des révolutions atlantiques puisqu'elle est constituée pour une bonne part d'exilés de la Révolution française. Dans les premières décennies de la république étastunienne, les flux sont impossibles à chiffrer mais concernent entre autres des missionnaires et des marchands. Les choses s'éclairent à partir des années 1840 : les Français constituent un flux ténu au sein d'un plus grand ensemble de migrations européennes en voie de massification.
Tous droits réservés @ Institut d'histoire de l’Amérique française, 2020

Ce document est protégé par la loi sur le droit d'auteur. L'utilisation des services d'Érudit (y compris la reproduction) est assujettie à sa politique d'utilisation que vous pouvez consulter en ligne.

https://apropos.erudit.org/fr/usagers/politique-dutilisation/ 


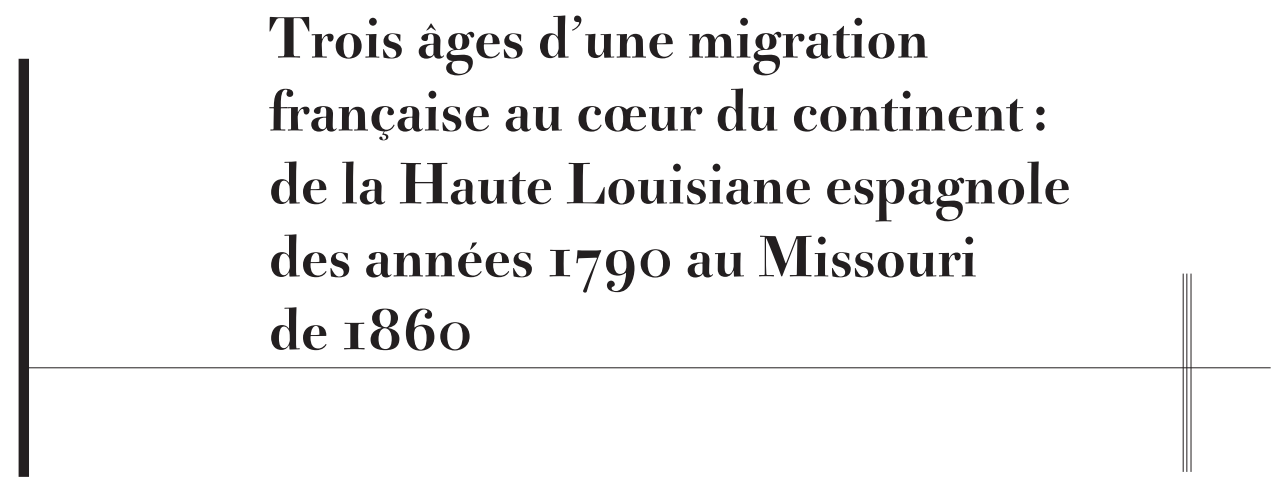

SOAZIG VILLERBU

Université de Limoges

RÉsumÉ • Les migrations françaises vers l'ancien Pays des Illinois ne s'arrêtent pas avec la fin du Régime français. Dans l'espace qui fut la Haute Louisiane espagnole avant de devenir l'État du Missouri, entre les années 1790 et 1860, cet article définit trois âges de cette migration. Elle est d'abord insérée dans l'histoire des révolutions atlantiques puisqu'elle est constituée pour une bonne part d'exilés de la Révolution française. Dans les premières décennies de la république étastunienne, les flux sont impossibles à chiffrer mais concernent entre autres des missionnaires et des marchands. Les choses s'éclairent à partir des années 1840: les Français constituent un flux ténu au sein d'un plus grand ensemble de migrations européennes en voie de massification.

ABstract - French migration to the former Illinois Country didn't not stop with the end of the French regime. In what was the Spanish Upper Louisiana before becoming the American state of Missouri, between the 1790s and 1860, this article defines three ages of this migration. It is first a small forgotten part the history of the Atlantic revolutions since it is mainly constituted of exiles of the French Revolution. In the first decades of the American sovereignty, the flow is impossible to quantify but concerns missionaries, merchants, and without doubt other ones difficult to discern. It is clearer from the 1840s: the French constitute a thin stream in the process of European migration's massification.

$\mathbf{L}$ e 24 novembre 1840, Nicholas Munsch déclare officiellement vouloir devenir citoyen américain. Né dans le Haut-Rhin, il est alors installé à Sainte-Geneviève, sur les rives du Mississippi au sud de Saint-Louis. Ses 
deux témoins, dans un processus qui le mène à la naturalisation en 1844, se nomment Anthony Mort et Joseph Grafton, deux anglophones. D'autres natifs de France entament la même démarche dans le même comté. Le 22 février 1845, par exemple, Jean Delmas est présenté par Barthélémy Ste-Gemme et Frédéric Chenu. Ste-Gemme est un Créole ${ }^{1}$, tandis que Chenu est présenté, tant lorsqu'il fait lui-même sa demande le 12 mai 1849 que lors du recensement fédéral de 1860, comme un Normand. Il a comme témoin le même Delmas et Isidore Dufresne, qui avait pour sa part déposé une demande identique le 29 juillet 1847. Douze ans plus tard, Damien André remplit le même formulaire et le dépose au responsable administratif du comté, Charles Rozier, Créole dont le père français était arrivé à Sainte-Geneviève en $1811^{2}$.

Nous avons là cinq hommes - aucune femme ne présente de demande individuelle dans ce corpus - nés en France, qui ont migré aux États-Unis, sont arrivés à Sainte-Geneviève et demandent à ne plus être Français mais Américains. Chacun a pris un chemin différent de socialisation ${ }^{3}$. Munsch l'Alsacien semble coupé de la francophonie: ses témoins sont anglophones et son épouse est née dans le grand-duché de Bade. Jean Delmas a pour sa part choisi un Français et un Créole pour témoins et a épousé une Créole de l'Illinois. Frédéric Chenu et Isidore Dufresne, eux, ont immigré aux États-Unis avec leurs épouses françaises. Damien André est célibataire; s'il est né en France, il n'a en 1859 que 22 ans. Or il est déjà présent au recensement de 1850, à 13 ans, avec sa famille dont tous les membres sont nés en France. Mais que signifiait pour lui cette naissance française quand sa jeunesse s'était écoulée sur les rives du Mississippi? Il avait face à lui au bureau du comté un cas miroir: Charles Rozier, né dans le Missouri d'un père français et d'une mère créole, Constance Roy, et qui épouse en 1859, l'année de la demande de Damien André, une autre Créole, Marie Émilie Lagrave.

En quelques brèves situations, les questions essentielles auxquelles je tenterai de répondre dans cet article sont posées. L'objectif est d’abord de définir, de compter et de localiser les migrants français à partir des années 1790 dans une région à l'identité mouvante: Haute Louisiane espagnole devenue française en titre seulement entre 1800 et 1803, puis étatsunienne

1. Dans le contexte missourien, un Créole est un Blanc francophone natif de la région, donc issu, au moins en partie, du premier peuplement colonial.

2. Missouri State Archives (MSA), St. Genevieve County Territorial Circuit Court, C 15672, déclarations d'intentions de demande de naturalisation.

3. J'utilise dans ce paragraphe les données issues du recensement fédéral de 1850, Volume Reel 0413 - 1850 Missouri Federal Population Census Free Schedule. 
et enfin, en 1821, État du Missouri jusqu’à la veille de la guerre de Sécession. On voit bien, à l'évocation de quelques destins individuels, à quel point la question de l'échelle d'observation est ici cruciale. On pourrait se contenter des quelques chiffres globaux accessibles à partir du recensement de 1850, le premier à être un tant soit peu précis sur l'origine géographique des habitants. En 1860, année terminale de cette étude, il y aurait 5283 personnes déclarant être nées en France et résidant dans le Missouri, soit une fraction résiduelle $(0,4 \%)$ de la population. Ce chiffre, pour utile qu'il soit puisqu'il permet de mesurer l'extrême faiblesse des flux migratoires français relativement à ceux provenant des États allemands ou d'Irlande, cache donc aussi une infinie variété de situations qui posent question: en quoi consiste une étude des «Français» de la région? Comment puis-je compter tous ceux qui sont désignés ou se considèrent comme tels?

Impossible évidemment de sonder les consciences en la matière, or on voit bien que sur le terrain rien n'est simple. Munsch l'Alsacien n'est peutêtre pas francophone et n'intègre donc pas les sphères a priori «françaises». D'autres sont venus de France, parlent français mais choisissent rapidement de ne plus être de nationalité française, tout en ayant des itinéraires matrimoniaux aussi variés que le sont les possibilités dans un Missouri dont l'histoire du peuplement colonial est complexe. D'autres encore n'entament pas de démarches administratives, mais sans que l'on puisse en savoir la raison: indifférence à la question, attachement au vieux pays? L'historiographie des migrations françaises en Amérique du Nord ne permet pas toujours de trancher; la plupart des rares travaux portant sur l'époque qui s'ouvre dans le dernier tiers du XIX ${ }^{\mathrm{e}}$ siècle, trop peu d'historiens se sont encore penchés sur les décennies antérieures. Ainsi, Jay Gitlin évoque les Français de la vallée du Mississippi mais sans chiffrer leur présence, et les seules analyses quantitatives d'ampleur portent sur la Californie, la Nouvelle-Orléans ou New York ${ }^{4}$. Dans ces quelques cas, c'est la faiblesse relative de la présence française qui fait son intérêt, car elle révèle une multitude de micro-réseaux et un éclatement des facteurs migratoires. Elle est donc propre à éclairer différemment l'ensemble des

4. Annick Foucrier, Le rêve californien. Migrants français sur la côte Pacifique (XVIII ${ }^{e}-X X^{e}$ siècles) (Paris, Belin, 1999); Jay Gitlin, Bourgeois Frontier. French Towns, French Traders and American Expansion (New Haven, Yale University Press, 2009); Marieke Polfliet, "Émigration et politisation: les Français de New York et La Nouvelle-Orléans dans la première moitié du XIX e siècle (1803-1860)", thèse de doctorat (histoire), Université de Nice-Sophia Antipolis, 2013; Malcolm J. Rohrbough, Rush to Gold: The French and the California Gold Rush, 1848-1854 (New Haven, Yale University Press, 2013). François Weil avait tenté une première synthèse dans «French Migration to the Americas in the 19th and 20th Centuries as a Historical Problem ", Studi Emigrazione, 123 (septembre 1996), p. 443-460. D’autres travaux seront cités au fur et à mesure de leur discussion. 
migrations atlantiques parfois écrasées sous les grands nombres et les généralisations.

In fine, ce qui sera analysé ici, c'est le destin de tous ceux qui se déclarent ou sont réputés nés en France et se sont installés dans la région, quels que soient leurs choix ultérieurs et justement parce que la variété de ces itinéraires et de ces choix éclaire des processus historiques qui se croisent dans le Missouri. Je traiterai de la question en trois séquences et sans prétendre épuiser le sujet. La première séquence relève des révolutions atlantiques. Ou plutôt, en l'espèce, de la contre-révolution. La rive droite du Mississippi, lorsqu'elle est espagnole, a le triple avantage pour certains d'être monarchiste, catholique et francophone. Elle est donc un refuge pour les exilés de la Révolution, havre certes extrêmement marginal d'un point de vue quantitatif mais tout à fait significatif de phénomènes de grande ampleur. Avec l'achat de la Louisiane par les États-Unis s'ouvre une nouvelle phase: des Français s'installent alors à la "confluence ${ }^{5}$ " précisément parce qu’elle est devenue étatsunienne, qu'elle semble ouvrir les promesses de la Révolution américaine et que de nouvelles occasions s'offrent donc à eux. Encore peu nombreux, ils s'inscrivent là encore dans des dynamiques qui les dépassent: ce sont des missionnaires appelés à construire l'Église catholique américaine, ce sont aussi des marchands qui veulent lier l'Atlantique et le cœur du continent. Ce sont bien d'autres encore, mais qui demeurent peu nombreux par rapport aux arrivées des années 1840 et 1850 . Une autre phase s'ouvre alors, celle de la massification des migrations ${ }^{6}$. Les Français y prennent part à leur échelle et la possible spécificité de leurs microsystèmes migratoires vers le Missouri s'efface dans un monde qui est celui, encore, des révolutions, mais aussi des nations et d'un nouveau capitalisme, autant de phénomènes que l'on doit penser à l'échelle globale et qui informent les destins migratoires à l'échelle locale.

\section{LA HAUTE LOUISIANE DE LA CONTRE-RÉVOLUTION}

L'historien des migrations doit pouvoir mettre en balance l'indispensable analyse quantitative et celle de destins individuels et familiaux. Mais il est des cas où la pesée globale est rendue pour le moins délicate par l’absence d'archives. La Haute Louisiane constitue un tel cas. Depuis 1763, le Pays

5. Stephen Aron, American Confluence: The Missouri Frontier from Borderland to Border State (Bloomington, Indiana University Press, 2006).

6. La périodisation de l'histoire des migrations considère de manière générale ces années 1840 comme tournant. Voir deux synthèses d'Adam McKeown, "Global Migration, 1846-1940", Journal of World History, 15, 2 (2004), p. 155-189 et «Les migrations internationales à l'ère de la mondialisation industrielle, 1840-1940», Le Mouvement Social, 241 (2012), p. 31-46. 
des Illinois des Français est devenu espagnol, comme, en théorie, l'ensemble de la vallée du Mississippi. Les autorités espagnoles n’ont pas cherché à y attirer des migrants de la péninsule ibérique ou de leurs possessions américaines et se sont contentées d'une armature administrative confiée la plupart du temps aux élites créoles, donc francophones. Si des chercheurs se sont penchés sur la période française ${ }^{7}$, peu ont encore travaillé les quatre décennies espagnoles, hormis Carl Ekberg ${ }^{8}$ et quelques analyses centrées sur Saint-Louis ${ }^{9}$. Mais trop rares sont les tentatives de véritable histoire sociale du peuplement colonial ou son analyse en termes de systèmes migratoires ${ }^{10}$. Jennifer Constantin l'a réalisé pour une partie de la population de Sainte-Geneviève, dont elle montre bien la mobilité spatiale et sociale à la fin du XVIII ${ }^{e}$ siècle, mais il reste beaucoup à faire ${ }^{11}$. Un des freins est le manque de sources à l'échelle régionale indiquant systématiquement les origines géographiques des habitants. Les autorités espagnoles ont bien mené des recensements, comme du reste l'Église catholique, mais ils sont tous très globaux; fondamentaux pour mesurer la population de la colonie, ils n'aident pas à comprendre les dynamiques du peuplement. Or des migrants continuent d'arriver de la vallée du SaintLaurent, les circulations sont permanentes au sein de la vallée du Mississippi, les populations se déplacent et fondent des villages; de plus, dans les années 1790, les Espagnols décident d'accueillir des colons étatsuniens en mouvement vers l'Ouest depuis déjà longtemps, de même qu'ils admettent quelques Français fuyant leur Révolution.

Le problème est bien la mesure précise de ces flux et de ces stocks. Les belles archives de la propriété foncière dans la région n'éclairent pas les origines autrement que par l'anthroponymie, aux limites bien connues. Deux documents permettent cependant de tenter d'y voir plus clair. Ils

7. Par exemple Cécile Vidal, «Les implantations françaises au Pays des Illinois (1699-1765)», Thèse de doctorat (histoire), EHESS, 1995; Robert K. Morrissey, Empire by Collaboration: Indians, Colonists, and Governments in Colonial Illinois Country (Philadelphie, University of Pennsylvania Press, 2015).

8. Carl J. Ekberg, Colonial Ste. Genevieve: An Adventure on the Mississippi Frontier (Carbondale, Southern Illinois University Press, 2014 [1985]); French Roots in the Illinois Country: The Mississippi Frontier in Colonial Times (Bloomington, University of Indiana Press, 1998); François Vallé and his World: Upper Louisiana before Lewis and Clark (Columbia, University of Missouri Press, 2002). Les travaux de cet auteur, s'ils sont utiles, souffrent de leur essentialisation d'une société française d'Ancien Régime qui se serait reproduite en Amérique.

9. Patricia Cleary, The World, the Flesh, and the Devil: A History of Colonial St. Louis (Columbia, University of Missouri Press, 2011).

10. Ce qui se lit dans les synthèses produites par Cécile Vidal, «La colonie du Mississippi» et «Le Pays des Illinois, 1673-1818», dans Yves Frenette, Étienne Rivard et Marc Saint-Hilaire, dir., Atlas historique du Québec. La Francophonie nord-américaine (Québec, Les Presses de l’Université Laval, 2012), p. 41-50 et p. 51-58.

11. Jennifer Constantin, «Canots, terres et fourrures en Haute-Louisiane: Les voyageurs de Ste-Geneviève convertis à la sédentarité (1763-1803)", mémoire de maîtrise (histoire), Université d’Ottawa, 2011. 
sont connus des historiens, mais n'ont jamais fait l'objet d'une analyse systématique. Ils ont été rédigés par deux Français, le père et le fils, au service de la Couronne d'Espagne. Ils constituent donc un regard spécifique et les catégories qu'ils utilisent ne sont pas nécessairement celles des acteurs sociaux de la région. Ils sont à la fois source et objet d'étude.

Pierre Charles de Hault de Lassus de Luzières est né en 1738 à Bouchain (dans l'actuel département du Nord) dont il est maire à la veille de la Révolution en plus de son poste de trésorier des États du Hainaut ${ }^{12}$. Désireux de fuir la France, il s'engage dans l'aventure de la colonie du Scioto, vaste spéculation financière atlantique sise dans le futur État de l'Ohio et par laquelle quelques aristocrates français espèrent une nouvelle vie américaine loin de la Révolution. L'aventure échoue et Lassus élabore un autre plan: réfugié à la Nouvelle-Orléans, il contracte avec les autorités espagnoles pour fonder une colonie française dans la Haute Louisiane en y attirant tous ceux qui, à la suite de l'aventure du Scioto, ont non loin de là fondé Gallipolis et y mènent une vie peu enviable. La Nouvelle-Bourbon (New Bourbon) est bien fondée au sud de Sainte-Geneviève, et devient le foyer des Lassus. Mais point de migrants français: l'opération est un échec, les habitants de Gallipolis deviennent des Américains du bassin de l'Ohio. Une deuxième tentative de colonisation a plus de succès et ce sont des Étatsuniens qui franchissent le Mississippi pour peupler le nouveau district de New Bourbon.

Or en 1797, Lassus réalise un recensement des trois pôles de peuplement européen que comprend son district ${ }^{13}$. Ce recensement est exceptionnel car, même s'il n'identifie précisément que les chefs de ménage (en listant par catégorie les autres personnes s'y rattachant), il est bien le seul à attribuer à chacun des 92 chefs de ménage un marqueur ethnoculturel et une identité religieuse.

À première vue, nous avons affaire à des communautés de taille réduite et de nature fort différente. La Saline et Bois-Brûlé sont des pôles de peuplement anglophone, avec dans le premier cas une minorité francophone et dans les deux cas une poignée de familles de Marylanders, des migrants étatsuniens catholiques ${ }^{14}$ qui auront du fait de leur foi des éléments à par-

12. Carl J. Ekberg, A French Aristocrat in the American West: The Shattered Dreams of De Lassus de Luzières, (Columbia, University of Missouri Press, 2011). Sur Gallipolis, voir Jocelyne Moreau-Zanelli, Gallipolis : histoire d'un mirage américain au XVIII' siècle (Paris, L'Harmattan, 2000).

13. Le recensement est transcrit dans C. J. Ekberg, A French Aristocrat..., p. 164-198, qui n'en fait pas l'analyse.

14. Thomas W. Spalding, C.F.X., "The Maryland Catholic Diaspora », U.S. Catholic Historian, 8, 3 (été 1989), p. 162-172; Maura Jane Farrelly, Papist Patriots: The Making of An American Catholic Identity (New York, Oxford University Press, 2012). 
Les chefs de famille du district de la Nouvelle-Bourbon en 1797

\begin{tabular}{|l|c|c|c|c|}
\hline \multicolumn{1}{|c|}{ Origine } & $\begin{array}{c}\text { La } \\
\text { Nouvelle-Bourbon }\end{array}$ & La Saline & Bois-Brûlé & Total \\
\hline Créole & 15 & 2 & & 17 \\
\hline Canadien & 9 & 3 & & 12 \\
\hline Français & 3 & 3 & & 6 \\
\hline Américain catholique & 4 & 3 & 1 & 8 \\
\hline Américain protestant & 3 & 28 & 10 & 41 \\
\hline Irlandais & 1 & 1 & 3 & 5 \\
\hline Écossais & & 1 & & 1 \\
\hline Allemand & 1 & & & 1 \\
\hline Total & $\mathbf{3 7}$ & $\mathbf{4 1}$ & $\mathbf{1 4}$ & $\mathbf{9 2}$ \\
\hline
\end{tabular}

Source: Carl J. Ekberg, A French Aristocrat in the American West:The Shattered Dreams of De Lassus de Luzières (Columbia, University of Missouri Press, 20I I), p. I64-198.

tager avec les Canadiens, Créoles et Français. Ces francophones se concentrent à la Nouvelle-Bourbon proprement dit, mais il faut ici questionner la présence de six chefs de famille qualifiés de Français à la Nouvelle-Bourbon comme à La Saline. Il s'agit de Lassus lui-même, de Nicolas Caillot dit Lachance, Louis Tonnelier, Henry Peyroux de la Coudrenière, monsieur Gensack et de Joseph Barbier. Je reviendrai sur chacun de ces hommes.

Le 6 mars 1804, c'est Charles du Hault de Lassus, plus connu sous le nom de Carlos Duhault Delassus, fils du fondateur de la Nouvelle-Bourbon, qui dresse une autre liste. Dernier gouverneur de la Haute Louisiane espagnole, il adresse à Amos Stoddart, qui a pris possession de la région au nom des États-Unis, un état des élites locales. Delassus rédige une sorte de commentaire qualitatif sur chaque homme qui possède selon lui une fraction du pouvoir politique, militaire ou religieux. Il veut indiquer à Stoddart sur qui s'appuyer afin d'assurer une sorte de continuité des structures de pouvoir. On trouve ainsi sur cette liste 41 hommes ${ }^{15}$. Deux sont clairement identifiés comme des Français. En tant que membres des élites coloniales, ils sont restés fameux: Antoine Soulard, «un ancien officier de la marine française emigré depuis la révolution», Jacques Marcellin Ceran Delassus St. Vrain, propre frère de Delassus, "Officier de la marine française emigré dans ce pays avec sa famille depuis 1797 ». Ce qui importe sans doute plus à Delassus

15. Missouri Historical Society (MHS), Delassus-St. Vrain collection, Box 2, folder 2, liste des principaux habitants de la colonie adressée à Amos Stoddard, 6 mars 1804. 


\section{Le recensement du district de la Nouvelle-Bourbon en 1797}

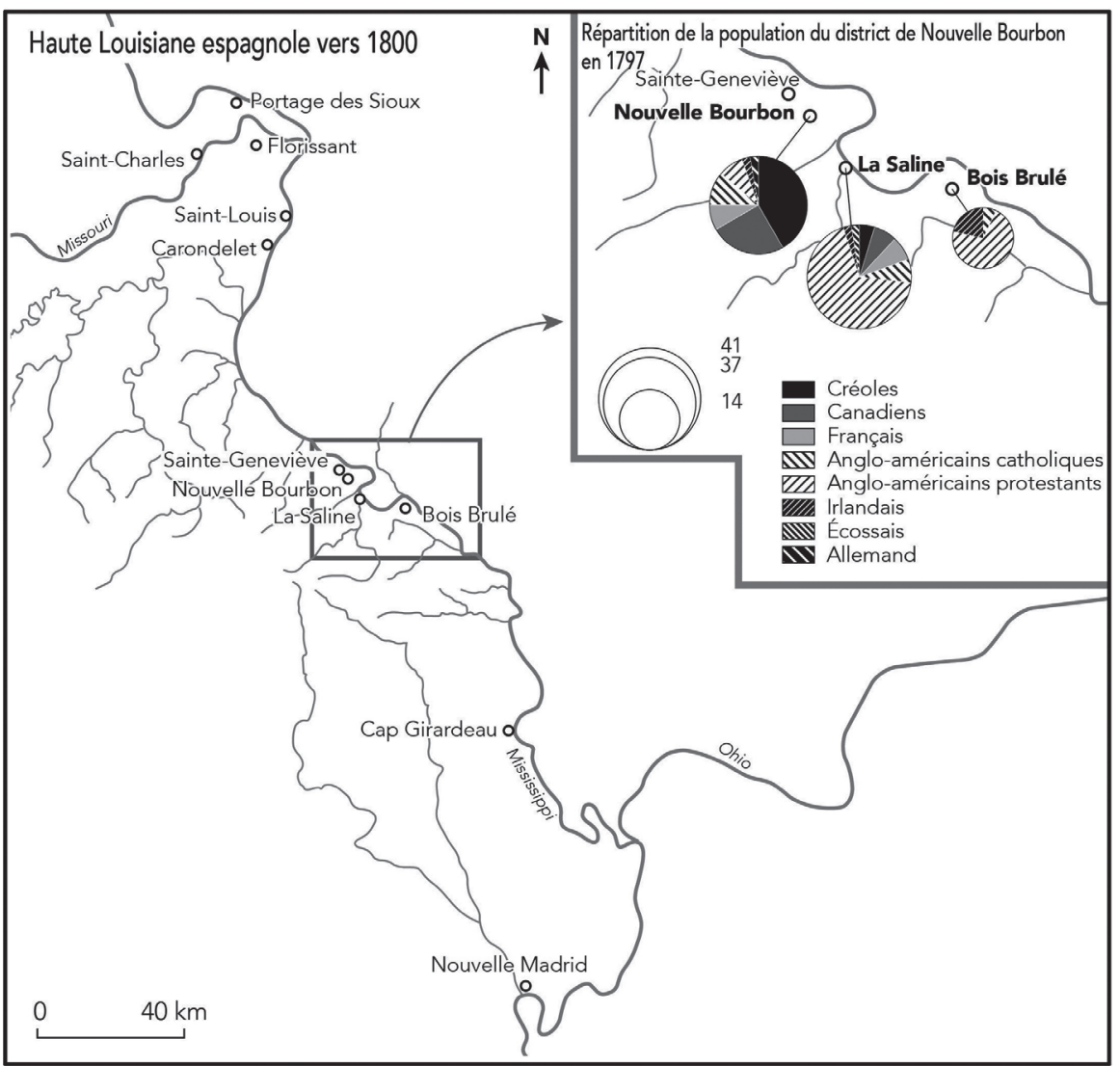

Conception Soazig Villerbu/Réalisation Pascal Brunello, CTIG, Université de La Rochelle

que leur qualité de Français, c'est leur carrière militaire, garantie de leurs compétences dans l'administration de la province. Car il n’identifie pas l'origine des autres Français de la liste. Leurs itinéraires sont pourtant connus. Il s'agit d'Antoine Saugrain, seul médecin de la colonie; des deux prêtres Pierre Jeannin (paroisse de Saint-Louis) et Léandre Lusson (paroisses de Saint-Charles et Saint-Ferdinand); de l’«officier zélé» Pierre Antoine La Forge; et enfin du frère (Camille, qui contrairement à St-Vrain n'était pas officier en France) et du père (Pierre) de Delassus.

Finalement sur les 41 hommes cités, 8 sont nés en France et arrivés très récemment dans la colonie. La proportion est de l'ordre de $20 \%$. Elle n'est absolument pas représentative de la situation démographique d'ensemble 
de la Haute Louisiane telle qu'on peut l'estimer, mais tient uniquement à la nature de la liste, qui met en évidence le caractère du flux ténu de migration française des années 1790 . Ces officiers, aristocrates, prêtres et médecins se retrouvent dans ces terres espagnoles loin de la Révolution et l'administration espagnole les a accueillis avec bienveillance. Delassus en donne en creux une raison. Les 33 autres administrateurs cités représentent en effet un conglomérat improbable de Créoles, Espagnols (les Vazquez père et fils), Britanniques ou États-Uniens dont la qualité, d'après Lassus, laisse parfois à désirer. Car à côté des incontestables Chouteau ou Vallé, trois de ces administrateurs sont analphabètes, deux ont d'importants problèmes d'alcool et la situation d'un Pierre La Pie est révélatrice des difficultés de la colonie: "Mauvais sujet mais le moins mauvais que j'ai trouvé et il me faloit absolument un sindic a marameck. quand je l'ai nommé il parle l'anglois. Homme determiné. » Qualités linguistiques et force de caractère suffisent donc à faire embaucher un "mauvais sujet» quand le bassin de recrutement est trop étroit.

Cela dit, Delassus oublie, volontairement ou non, quelques Français connus par ailleurs. Auguste Charles Frémon de Laurière et Louis Auguste Tarteron de la Beaume avaient a priori le profil idéal pour être inclus dans sa liste, mais n'ont pas participé à l'administration de la colonie, préférant faire des affaires, seuls ou avec des associés ${ }^{16}$. Pas plus que Jacques Guibourd et Pierre Provenchère qui, lorsque Delassus passe le flambeau à Stoddart, ont tout juste mis fin à leur partenariat dans une boutique de Sainte-Geneviève ${ }^{17}$. Il manque également le marchand Mathurin Michel Amoureux, qui vient de s'installer à La Nouvelle-Madrid en venant du Kentucky ${ }^{18}$. Ces commerçants ne correspondaient pas aux objectifs du gouverneur, qui ne comptaient pas sur eux dans l'établissement et le maintien du pouvoir politique et religieux.

Que conclure de l'analyse rapide de ces deux sources? D’abord que ces quelques éléments sont incomplets du fait de leurs limites spatiales, sociales et genrées. Il manque ici la masse de la population de la colonie, ces fermiers et ces coureurs des bois - souvent les mêmes, pratiquant une double activité - dont on suppose que la plupart sont venus (eux ou leurs

16. Ils font partie de la petite poignée de Français mentionnés à titre d'anecdotes par Charles Van Ravenswaay dans St. Louis: An Informal History of the City and Its People, 1764-1865 (Saint-Louis, Missouri Historical Society Press, 1991), p. 113-114.

17. Le livre de compte de leur association demeure une mine pour l'historien: MHS, Louis Guibord Collection, livre de compte 30 novembre 1799-14 avril 1801.

18. Soazig Villerbu, "Sainte-Geneviève (Missouri) et le monde atlantique: Michel Amoureux en 1813 », <https:// corridor.hypotheses.org/58>. 
parents) de la vallée du Saint-Laurent ${ }^{19}$, bien que quelques individus nés en France puissent se retrouver parmi eux. Or il n'existe pas d'étude systématique sur le sujet et les généalogistes, indispensables dans ce genre de situation, n'ont pas traité l'ensemble des familles des époques française et espagnole. Mais il manque aussi les femmes puisque ni le père ni le fils Lassus ne donnent de précisions sur elles. Or certains de ces migrants sont venus sur les rives du Mississippi avec leur épouse née également en France. C'est le cas de Pierre du Hault de Lassus, mais aussi, par exemple, de Michel Amoureux dont on sait que l'épouse, «la citoyenne janvier», était en France une «excellente patriote» comme son mari qu'elle rejoint en Amérique ${ }^{20}$. Mais pour l'une dont quelques indices subsistent, combien de femmes invisibilisées par les discours de l'époque et les pratiques archivistiques ultérieures?

Malgré tout, il faut tenter une typologie sur la base de ces quelques éléments. Et il est d'abord nécessaire de dégager des cas particuliers. Si Nicolas Caillot dit Lachance est bien né en France en 1733, il est arrivé au Pays des Illinois comme charpentier sous le Régime français et a épousé Marianne Giard à Kaskaskia en 1757 avant de s'installer tardivement sur la rive espagnole, où il décède en $1799^{21}$. Il n'entre donc pas dans le champ de cette étude mais montre qu'il peut y avoir d'autres cas comme le sien, sorte de témoignages ultimes de la colonisation française. Il faut écarter également Gensack et Barbier, de la Nouvelle-Bourbon, sur qui on ne sait rien et pas même leur date d'arrivée. Il reste les autres, que l'on peut classer, avec toutes les nuances inhérentes à ce genre d'entreprise. Deux hommes, d'abord, sont arrivés depuis la Louisiane espagnole: Henri Peyroux de la Coudrèniere, venu au printemps 1785, avec 168 Acadiens $^{22}$; il s'y était fait le vain zélateur d'une immigration venue de France (et indirectement d'Acadie) organisée et massive, avant de s'installer à Sainte-Geneviève à la fin des années 1780; tandis que Louis Tonnelier est un jeune Parisien éduqué qui migre en Louisiane espagnole puis se marie à Sainte-Geneviève en 1786 et y devient temporairement maître d'école l'année suivante ${ }^{23}$.

Les autres - il s'agit toujours de ces élites discernables dans les sources sont pour la plupart venus en Haute-Louisiane pour échapper à la

19. Le fait est avéré à Sainte-Geneviève, voir J. Constantin, "Canots, terres... ».

20. MHS, Amoureux-Bolduc papers, Box 1, folder 1, certificat octroyé par la municipalité de Bourgneuf le 30 prairial an 2 (18 juin 1794).

21. Voici un cas pour lequel les travaux d'érudition locale sont nécessaires: <http://mjgen.com/ colyott/manuscript.html>.

22. Archives départementales de Loire-Atlantique, C1287, rôle d'armement du Beaumont 12 mai 1785.

23. C. J. Ekberg, Colonial Ste Genevieve..., p. 348-356 pour Peyroux et p. 275-276 pour Tonnelier. 
Révolution française. Ce sont des émigrés et des réfractaires, ces prêtres ayant refusé le serment à la Constitution Civile du clergé de 1790. Parmi les premiers on compte la famille de Luzières bien sûr. Mais aussi celles de Pierre Antoine La Forge, de Frémon de Laurière, de Soulard, de Tarteron de la Beaume ou de Jacques Guibourd (ce dernier aussi réfugié de Saint-Domingue) ou encore celle de Pierre Provenchère (attaché à la cour de Louis $\mathrm{XVI}^{24}$ ) ainsi que Les Luzières fils et les Laforge qui chevauchent un second groupe, celui des réfugiés de Gallipolis. Leur premier exil était en effet aux États-Unis, mais son échec les a menés plus loin encore et finalement à une plus grande cohérence idéologique. Ils côtoient Antoine Saugrain qui, lui, était arrivé au même projet par un goût de l'Amérique qui lui a fait entrecroiser des réseaux parfois contradictoires. Il n'est arrivé à Saint-Louis qu'en 1800 après quelques années à Lexington, dans le Kentucky, à la suite de l'échec de Gallipolis ${ }^{25}$.

Auprès d'eux, et qu'ils connaissent sans aucun doute, opèrent des prêtres qu'il faut replacer dans un ensemble plus large. Le clergé catholique clairsemé du Pays des Illinois à l'époque espagnole est d'une extraordinaire variété. Quelques Français sont notables. Jean-Antoine Le Dru témoigne jusqu'à son décès, dans les années 1790, de l'ancienneté de l'implantation française, mais d'autres se déploient dans la région comme dans un refuge. Léandre Lusson et Pierre Jannin sont bien des réfractaires. Le deuxième avait été embauché à la fois par $\mathrm{M}^{\mathrm{gr}}$ Carroll, évêque de Baltimore, et l'État fédéral, pour être prêtre et agent indien aux États-Unis avant qu'il ne s'installe sur une rive espagnole, région sans aucun doute plus conforme à ses convictions ${ }^{26}$.

Reste l'exception: Michel Amoureux. Né à Bourgneuf en 1747, marchand à Lorient impliqué dans le commerce avec les États-Unis pendant la guerre

24. La notice de la MHS, Provenchere family papers, le donne comme «tuteur du Comte d'Artois» sur la foi sans doute d'une lettre de Pierre Provenchère fils (MHS, Provenchère fils à Provenchère père, 28 juillet 1814), mais il vaut mieux y voir le premier valet de chambre du duc de Berry (Charles-Ferdinand d'Artois, deuxième fils du comte d'Artois futur Charles X) à partir de 1784 (d'après Mathieu da Vinha, communication personnelle du 24 octobre 2016).

25. Saugrain mériterait qu'un historien se penche sur son cas en renouvelant quelques travaux anciens comme celui d'Hélène Fouré-Selter, L'Odyssée américaine d'une famille française. Le docteur Antoine Saugrain (Baltimore, Johns Hopkins University Press, 1936). Voir aussi J. Moreau-Zanelli, Gallipolis..., p. 71-74.

26. Pour le cadre général, voir Tangi Villerbu, «Structurer un territoire ecclésiastique: la géopolitique catholique entre Grands Lacs et Mississippi (1763-1803)", dans Laurent Veyssière, Philippe Joutard et Didier Poton, dir., Vers un nouveau monde atlantique : Les traités de Paris, 1763-1783 (Rennes, Presses universitaires de Rennes, 2016), p. 211-221. Et pour Jannin, Tangi Villerbu, «Vincennes (Indiana), 1795-1804: convertir ou conserver? Le travail du père Rivet», dans Pierre Ragon, dir., Nouveaux chrétiens, nouvelles chrétientés dans les Amériques (XVI $I^{e}$ XIX $X^{e}$ siècles) (Nanterre, Presses universitaires de Paris Ouest, 2014), p. $277-300$. 
d'Indépendance. Partisan de la Révolution, il quitte la France en 1793 parce que les Vendéens ont brûlé ses biens et non parce qu'il les soutient. Il s'installe d'abord à Georgetown où sa famille le rejoint, puis dans le Kentucky. Son arrivée à New Madrid est finalement énigmatique, comme s'il avait anticipé la transmission de la Louisiane à la France prévue secrètement par le traité de San Ildefonso en 1800. Dans tous les cas, Amoureux se réjouissait de repasser sous l'autorité française ${ }^{27}$. Ce fut vain puisque les États-Unis prennent, en fait, possession du territoire. Qu'importe: Amoureux réintègre avec plaisir la République qu'il a quittée récemment et dénonce d'un même geste des Créoles ignorants et des Européens intrigants à l'esprit jésuite ${ }^{28}$. On comprend que Delassus ne l'ait pas inclus dans sa liste: l'incompatibilité idéologique était en fait totale.

Ce que révèle cette situation des Français de Haute Louisiane au moment de la passation de pouvoir, c'est à quel point l'histoire de la région est indissociable de l'histoire atlantique. Si Amoureux est exclu du champ du gouverneur, c'est que leurs visions de la Révolution française et du monde en général ne concordent pas. Quelle importance, a priori, sur les rives du Mississippi ? En fait la région est connectée par ses élites - et il resterait à trouver le moyen d'approcher le grand nombre - aux flux démographiques, culturels, politiques qui agitent le temps des révolutions. Elle peut faire figure de refuge isolé alors qu’elle est en fait un élément, certes lointain, d'un monde atlantique avec lequel elle vibre à son propre rythme en prouvant que les intuitions de François Furstenberg peuvent être prolongées au-delà encore de l'Ouest transappalachien. Le cœur du continent est atlantique et travailler les migrations françaises au temps des révolutions est un excellent moyen d'accéder à des connexions imprévues mais bien réelles ${ }^{29}$.

\section{I803-1840. ÉTATS-UNIS, AFFAIRES ET MISSIONNAIRES}

Joseph Garnier est un réfugié de Saint-Domingue - il y avait migré très tardivement, en 1791 - parmi les milliers qui se sont installés aux États-

27. Historic New Orleans Collection, Pierre-Clément Laussat papers, MSS 125.234, Mathurin Michel Amoureux à Pierre-Clément Laussat, 3 août 1803.

28. Mathurin Michel Amoureux à Albert Gallatin, 2 novembre 1804, dans Clarence Edwin Carter, The Territorial papers of the United States, vol. XIII, The Territory of Louisiana-Missouri, 1803-1806 (Washington, US printing Office, 1948), p. 64-67.

29. François Furstenberg, "The Significance of the Trans-Appalachian Frontier in American History", American Historical Review, 113 (juin 2008), p. 647-677; et When the United States Spoke French: Five Refugees Who Shaped a Nation (New York, Penguin Press, 2014). 
Unis dans les années $1790^{30}$. Il mène ses affaires depuis New York lorsque la Louisiane devient américaine en 1803. Il y voit rapidement une belle occasion et il y organise le transfert de ses activités. Arrivé à Saint-Louis début août 1805 , il décrit à sa sœur un

pays neuf presque désert etant entouré de sauvages de touttes nations et les ayant presque presque tous les jours parmi nous, c'est pourtant un pays susceptible de devenir par la suite un des premiers pays du monde comme les terres y étant de toute excellence et le pays abondant en mines de toutes espèces. L'espoir dy faire de l'argent et non la beauté présente my a conduit et j'espere que cet espoir se réaliser sous peu ${ }^{31}$.

\section{La nouveauté est frappante mais nuancée:}

J'ai ici pour ami un Monsieur Soulard de Rochefort dont les parents sont encore vivants dans ce pays la et lui écrivent très souvent, tu pourrais profiter des mêmes occasions - Soulard est un ancien officier de marine $\&$ un très charmant garçon - il est marié ici a une Créole du pays $\&$ assez a son aise Nous parlons très souvent de notre pays - Il y aussi un docteur Saugrain de Paris dont la sœur est mariée a notre parent (éloigné à la verité) le médecin Guillotin $^{32}$.

À première vue, le départ de Garnier, pour ce qui n'est pas encore le Missouri mais toujours la Louisiane, marque une étape importante. S’il quitte la côte Est, c'est parce que la région est tout juste devenue étatsunienne et qu'elle est, de ce fait, recouverte par un nouveau récit, celui de l'expansion nationale vers l'Ouest. Au-delà du Mississippi, donc, ce n'est pas une vieille colonie que devait atteindre Garnier, mais un pays neuf, sauvage, où la culture américaine du profit et de l'ambition individuelle pouvait se déployer sans borne ${ }^{33}$. La région de Saint-Louis exemplifierait alors ce destin étatsunien. Pour autant, on voit bien que l'effacement discursif du passé

30. Pour la Louisiane, voir Nathalie Dessens, From Saint-Domingue to New Orleans: Migration and Influences (Gainesville, University Press of Florida, 2007) et Creole City: A Chronicle of Early American New Orleans (Gainesville, University Press of Florida, 2015). Une vue d'ensemble dans R. Darrell Meadows, «Engineering Exile/Social Networks and the French Atlantic Community, 1789-1809 ", French Historical Studies, 23,1 (2000), p. 67-102. Il existe une petite poignée de réfugiés de Saint-Domingue dans le Missouri, qui s'y sont parfois installés tardivement après de longs séjours à l'Est ou ailleurs. Le Missouri est peutêtre terre d'accueil, mais bien résiduelle par rapport à la Louisiane.

31. Archives départementales de la Charente-Maritime (ADCM), 244 J 23, Joseph Garnier à sa sœur Marie-Elisabeth Garnier Polony, 9 septembre 1805.

32. Il s'agit du réformateur de la peine capitale dont la guillotine tire son nom. ADCM, $244 \mathrm{~J} 23$, Joseph Garnier à sa sœur Marie-Elisabeth Garnier Polony, 4 octobre 1806.

33. Joyce Appleby, Inheriting the Revolution: The First Generation of Americans (Cambridge, The Belknap Press of Harvard University Press, 2000); Christopher Clark, Social Change in America from the Revolution through the Civil War (Chicago, Ivan R. Dee, 2006); J. M. Opal, Beyond the Farm: National Ambitions in Rural New England (Philadelphie, University of Pennsylvania Press, 2008). 
franco-espagnol au profit d'un pré-discours de la frontière est rapidement atténué par la réalité. À Saint-Louis, il retrouve Antoine Soulard, vieil ami de la famille et issu de la même vallée de la Charente, et Antoine Saugrain, cousin éloigné.

On a là, finalement, les deux faces de la région telle qu'envisagée et vécue par les migrants à partir de 1803. Elle est à la fois terre de conquête et de colonisation de peuplement étatsunienne et ancienne terre française où la créolité, pour quelques décennies encore, dessine un paysage humain particulier qui explique des itinéraires migratoires spécifiques. Elle explique aussi la force de l'enracinement des Français arrivés dans les années 1790 et qui, bien qu'ils aient fui la Révolution, ne cherchent pas à regagner leur pays d'origine sous l'Empire ou la Restauration. Les alliances économiques ou matrimoniales avec les familles créoles dessinent des réseaux suffisamment denses pour que l'installation soit définitive. Les célibataires prennent épouse sur place, comme le feront leurs fils. St. Vrain, par exemple, épouse Marie Félicité Dubreuil, tandis qu’Eugène Guibourd, le fils de Jacques, épouse Ursule Barbeau. Pierre Provenchère fils s'est, quant à lui, uni à une famille hollandaise, les Rutgers. Mais ses témoins de mariage sont bien un émigré (Luzières) et un Créole (François Vallé, commandant du poste de Sainte-Geneviève) $)^{34}$. Lors de la séquence $1814-1815$, il se pose d'ailleurs la question du retour, comme il l'avait fait après le coup d'État de Bonaparte en 1799. Ses sentiments semblent clairs:

En arrivant à St Louis j'y ai appris l'heureuse révolution qui vient d'arriver en France a laquelle je ne m'attendois point et que je ne puis réellement comprendre mais dont pourtant nous ne devons pas douter unissons nous, mon tendre père, pour remercier l'être suprême de ce qu'il a exaucé nos prières et reconnaissons sa toute puissance. dans un evenement si peu probable et que l'Europe entière n'auroit pu produire si elle n'avoit été l'instrument de la justice Divine, voici donc notre patrie après 25 ans d'égarement et de malheurs renud a elle même, rendue a la paix, et dans la possibilité sous une administration sage de réparer les pertes en tous genres dont elle a été de si long tems victime, voici donc notre Roy, nos princes après 25 ans d'adversité, d'exil, d'humiliation, remontant sur le throne de leurs ancestres se trouvant au milieu des françois fidels, il m'est impossible de vous rendre tous les sentiments dont mon cœur est plein ${ }^{35}$.

34. Il tient à le rappeler quand il narre ses mésaventures commerciales à son père, MHS, Provenchère papers, Provenchère fils à Provenchère père, 2 février 1814.

35. MHS, Provenchère papers, Provenchère fils à Provenchère père, 28 juillet 1814 . 
Sans ambigüité, sous la République américaine, le voilà qui fantasme le retour des Bourbons. D’ailleurs, il "préfère vivre sous le despotisme d'un prince de la maison de Bourbon que dans ce pays de liberté ${ }^{36} »$. Mais dans le même mouvement, alors qu'il comprendrait que son vieux père n'ait plus rien à attendre de la vie que de revoir les princes du Sang à qui il était attaché en 1789, lui-même ne quittera pas cette terre américaine qu'il dit ne pas aimer; sa famille et ses affaires sont dorénavant trop enracinées dans la basse vallée du Missouri, en amont de Saint-Louis.

Les «anciens» sont donc restés, avec plus ou moins d’appétence pour le nouveau modèle américain qui leur est imposé - mais dans l'ensemble, comme membres des élites locales, ils passent le cap sans soucis - et ils sont rejoints par d'autres Français. Tenter une pesée globale de ces arrivées est sans doute encore plus difficile que pour les dernières années du régime espagnol. Le recensement fédéral américain ne comporte pas de données individuelles avant 1850 et il n'y a pas dans le Missouri (créé comme État en 1821) les sources utilisées par Marieke Polfliet pour travailler sur les communautés françaises de La Nouvelle-Orléans et de New York ${ }^{37}$. Le recensement de 1820 compte 63817 habitants dans le futur État et donne 496 «étrangers non naturalisés», ce qui indique en partie la faiblesse des flux non états-uniens: sans le comté de Saint-Louis, où $70 \%$ de ces étrangers sont concentrés, ils ne compteraient que pour $0,3 \%$ de la population. De plus ces données empêchent d'observer une part conséquente des arrivées: les migrants qui auraient fait le choix d'une naturalisation rapide.

Faute de données quantitatives complètes, mais en étant conscient que le flux de migrants français demeure ténu - quelques centaines d'individus tout au plus jusqu'aux années 1830 - il faut se résoudre à ne définir que quelques situations types. Faisons d'abord la part de cas limites: des natifs de France déjà fortement intégrés aux États-Unis et qui viennent dans le Missouri sans que leur qualité de Français soit particulièrement notée et notable. Jean-Baptiste (John Baptist) Lucas est dans ce $\operatorname{cas}^{38}$. Ce juriste normand faisait partie du cercle de Benjamin Franklin lorsque celui-ci était en poste en France et, séduit par le projet républicain des États-Unis, il y arrive en 1784. Sa carrière est si belle qu'il est nommé en 1805 juge du Territoire de Louisiane à Saint-Louis, chargé d’y installer le système judiciaire américain, celui, somme toute, de son pays. Sur place, il ne semble pas qu'il

36. MHS, Provenchère papers, Provenchère fils à Provenchère père, 22 janvier 1815 .

37. M. Polfliet, «Émigration et politisation...».

38. John Francis McDermott, «John B. C. Lucas in Pennsylvania», Western Pennsylvania Historical Magazine, 21 (septembre 1938), p. 209-230. 
s'associe particulièrement aux réseaux créoles, mais il rejoint par un biais la francophonie locale: homme des Lumières en France, il tient pourtant au lien avec le clergé catholique réfractaire dans le Missouri. Son fils James est envoyé au Collège St. Thomas, dans le Kentucky, grâce aux liens personnels tissés avec $\mathrm{M}^{\text {gr }}$ Flaget, l'évêque de Bardstown, et sa fille Anne ne tarit pas d'éloges sur le nouvel évêque de La Nouvelle-Orléans, $\mathrm{M}^{\mathrm{gr}}$ Dubourg ${ }^{39}$. C'est d'ailleurs dans ce cadre catholique uniquement que l'on trouve dans les papiers Lucas de la correspondance en français, comme s'il s'agissait là du dernier lien qui unissait la famille au monde francophone.

Lucas n'est sans doute pas seul dans son cas, mais les archives n'en disent rien. Elles se révèlent trop peu loquaces également, comme lors de la période précédente, sur d'autres Français, de classes sociales plus modestes que les Lucas et qui auraient pu immigrer pour participer aux activités économiques de base du territoire, parmi lesquelles le commerce des fourrures joue encore un rôle important. Et pourtant, ils existent et le rythme de leurs migrations ne s'expliquerait pas nécessairement par celui des soubresauts politiques français. L'un d'entre eux témoigne de courants souterrains à découvrir. Jean Brunet, avant le début des années 1820, demeure pourtant bien énigmatique : il est venu de France à Saint-Louis, sans doute en 1818, et est au service de Chouteau dans la haute vallée du Mississippi. Il travaille donc dans les pelleteries. Mais son parcours rapide vers la petite notabilité dans le Wisconsin laisse ouverte la question de ses origines sociales. Or on ne sait rien de précis de ses date et lieu de naissance, quelque part dans le Sud-Ouest dans les années 1790: est-il un homme du peuple ayant franchi l'océan puis le continent pour monter socialement, ou le fils d'une bonne famille atlantique bordelaise ${ }^{40}$ ?

De ce cas, finalement, il ressortirait d'une autre catégorie, celle des marchands qui perpétuent, en le modulant en fonction du contexte, un système atlantique bien rodé. Là encore, un recensement complet serait le bienvenu, sans doute des noms apparaîtraient qui ont échappé jusque-là au travail des historiens. Ferdinand Rozier peut servir ici, rapidement, de guide ${ }^{41}$. Issu de la bourgeoisie marchande nantaise, Rozier est envoyé aux États-Unis par

39. MHS, John B. C. Lucas papers, Box 7, Mgr Flaget à John B. C. Lucas, 20 novembre 1816; James Lucas à James B. C. Lucas, 9 décembre 1816; Box 8, Anne Hunt à John B. C. Lucas, 5 et 18 janvier 1818.

40. J’ai traité de Brunet dans le Wisconsin dans «La religion des bûcherons: Lucien Galtier dans la vallée de la Chippewa, juin 1842», Histoire \& Missions chrétiennes, 17 (mars 2011), p. 83-105. Mais le dossier serait à creuser vers l'amont. Brunet est-il ce jeune homme de 13 ans qui, le 9 mars 1810, obtient sous ce nom un passeport à Bordeaux pour rejoindre son père à Philadelphie? Archives départementales de la Gironde, 4M 687/148, <http://gael.gironde.fr/passeports-search-form.html>.

41. Je résume ici brièvement Tangi Villerbu, «Réseaux marchands et chaines migratoires. Entre Nantes et la vallée du Mississippi, fin 18 $8^{\mathrm{e}}$-début $19^{\mathrm{e}}$ siècle ", Enquêtes et documents, 54 (2016), p. 61-80 et 
son père en 1806, en compagnie de Jean-Jacques Audubon, avec qui il est associé pour exploiter de chimériques mines de plomb autour de Philadelphie ou, en cas d'échec, se tourner vers toute autre occasion commerciale. Les deux jeunes hommes sont donc partis vers l'Ouest, ouvrant boutique à Louisville puis à Shippingsport dans le Kentucky, avant de poursuivre jusqu'à Sainte-Geneviève en 1810. Là, leurs destins divergent dès 1811. Audubon brise l'association et vogue vers son propre destin, tandis que Rozier s'établit comme marchand et épouse Constance Roy. Celle-ci est apparentée aux Vallé, d'une part, et est la nièce d'Ursule Barbeau, d'autre part, ce qui fait de Rozier un membre du réseau créole le plus important de la place et un cousin d'Eugène Guibourd, fils de Jacques, l'émigré des années 1790. Cette sorte de fusion des élites francophones joue à plein à la génération suivante également puisque Ferdinand Rozier et Constance Roy, qui ont eu dix enfants, ont marié six de leurs garçons à des Créoles, Vallé en ligne directe ou cousines des Vallé. Deux autres garçons (l'aîné et le plus jeune) ont épousé, eux, des Anglo-Américaines, tandis que les deux filles se sont unies pour l'une à un Allemand, dans un mariage auquel ses parents se sont opposés et qui a mal fini; et pour l'autre à Charles Hertich, fils de Mecalite Villars, une Créole, et de Joseph Hertich, un Suisse qui était arrivé aux États-Unis en 1796 et à Sainte-Geneviève autour de 1810. Il faut y voir la spécificité de la migration française dans le Missouri: elle peut investir des réseaux de parenté (réelle ou spirituelle, car les structures de parrainage recoupent celles des mariages) anciennement francophones, créoles, français ou bien suisses, tout en jouant à la marge sur l'alliance avec les vrais nouveaux venus, ces Anglo-Américains jamais majoritaires à SainteGeneviève avant la guerre de Sécession mais qui, ailleurs, font valoir rapidement leur domination dans le processus de peuplement.

Pour autant des stratégies différentes sont possibles. Ainsi Firmin Desloges, le neveu de Ferdinand Rozier, franchit lui aussi l'océan, en 1823, pour s'associer à son oncle et tient boutique, pour sa part, dans les monts Ozarks, à Potosi, où la francophonie est tôt devenue minoritaire. Or il fait un choix opposé à celui de son oncle en demandant très vite la nationalité américaine et en épousant, en 1832, Cynthia McIlvaine, une AngloAméricaine protestante ne parlant pas un mot de français - et leurs enfants seront élevés en anglais. La question est épineuse: depuis la France, la famille de Firmin (et surtout sa mère, et sœur de Ferdinand) peste et s'in- 
quiète: comment communiquer avec Cynthia, d'une part, et comment envisager l'avenir des enfants si leur mère n'est pas catholique, d'autre part? Firmin se voudra toujours rassurant sur cet aspect: comme les Lucas, il tient à maintenir la foi comme lien communautaire ${ }^{42}$.

S’il peut le faire, c'est que l'Église a mené un vaste effort de construction d'un réseau paroissial et d'éducation. Encore largement imparfait dans les années 1830, il témoigne néanmoins d'un triple mouvement: la structuration par Rome des espaces de colonisation; le renouveau missionnaire de l'Europe des Restaurations ; la volonté des catholiques eux-mêmes de bénéficier d'infrastructures ecclésiastiques, non sans tensions avec la hiérarchie. Or le deuxième de ces mouvements suppose des migrations. En effet, l'arrivée de séminaristes, de prêtres et de sœurs doit être comprise comme un mouvement migratoire, qui fonctionne comme une chaîne institutionnelle forte, mais n'en met pas moins en branle des hommes et des femmes qui franchissent l'océan pour trouver un épanouissement professionnel aux États-Unis (et au Canada, car le mouvement doit être pensé à l'échelle nordaméricaine), comme tous les migrants ${ }^{43}$.

Or ces migrations sont à la fois fameuses et peu connues. Elles sont fameuses car l'Église entretient encore le culte de ses fondateurs: les premiers évêques (Dubourg, Rosati), les célébrités passées par le Missouri (Odin, Timon) ou les Sœurs de Saint-Joseph, collectivement (et donc anonymement) ou en vantant la figure première de Philippine Duchesne, canonisée en 1988. Mais elles demeurent peu connues. D’abord parce que trop peu d'historiens les considèrent comme des migrations. Ensuite parce l'historiographie du catholicisme américain demeure encore trop proche de l'institution ecclésiastique elle-même, ce qui nuit souvent à un regard neuf et critique sur le sujet. Paradoxalement - car c'est bien le seul domaine où cela se produit - les plus connues sont les femmes, du fait d'un fort investissement dans une histoire des religieuses depuis les années 1990. Il s'agissait de redécouvrir les logiques genrées à l'œuvre dans le catholicisme et de montrer les congrégations religieuses comme des ins-

42. MHS, Joseph Desloges collection, vol. 3, par exemple Mme Desloges à Firmin Desloges, $1^{\text {er }}$ juin 1832; Joseph Desloges à Firmin Desloges, 15 juin 1832 et 5 juillet 1832; Firmin Desloges à Mme Desloges, $1^{\mathrm{er}}$ novembre 1832 .

43. Je me permets de renvoyer à Tangi Villerbu, «Ramener une colonie de bons missionnaires. Le recrutement de prêtres européens pour les États-Unis au $19^{\text {ème }}$ siècle », Revue d'histoire moderne et contemporaine, 56-3 (2009), p. 33-65. Voir aussi Simon Balloud, «Les hommes d’Église français dans la migration vers le Canada, 1842-1914 », thèse de doctorat (histoire), Université de La Rochelle/Université du Québec à Montréal, 2018. 
titutions de pouvoir féminin ${ }^{44}$. Dans le Missouri, cela mène naturellement à l'analyse des Sœurs de Saint-Joseph, que Philippine Duchesne a amenées de France en 1818. Quatre Françaises l'accompagnaient - Octavie Berthold, Eugénie Audé, Catherine Lamarre et Marguerite Manteau - et d'autres devaient suivre. On connaît désormais bien leur travail éducatif auprès des jeunes filles de Saint-Louis et de ses environs ${ }^{45}$, leurs missions indiennes également, de même que les écrits de Philippine Duchesne qui sont maintenant intégralement publiés ${ }^{46}$. Pour autant, dans une logique d'analyse des migrations, il reste à faire. En effet, aucune étude n'a encore été menée sur les religieuses comme groupe migrant: nous ne disposons pas de données chiffrées précises, pas plus que des biographies qui permettraient de comprendre les structures de la migration.

Il en va de même pour les hommes : les missionnaires européens abondent dans le Missouri, mais leur connaissance demeure strictement narrative, sans aucune tentative quantitative ni structurelle. Il faudrait, pour parvenir à un tableau complet, réaliser une prosopographie des prêtres du diocèse de Saint-Louis. Une source permet d'y voir plus clair, au moins au sujet des séminaristes. L'établissement de Sainte-Marie des Barrens a été fondé en 1818 à Perryville et confié aux Lazaristes que $\mathrm{M}^{\mathrm{gr}}$ Dubourg venait de recruter dans le Royaume de Naples. Richard Janet en a tracé utilement l'histoire, mais sans jamais utiliser de données chiffrées ${ }^{47}$. Or pour la période 1818-1828, il existe un registre des élèves.

44. La bibliographie est immense. Voir notamment Anne M. Butler, Across God's Frontiers : Catholic Sisters in the American West, 1850-1920 (Chapel Hill, University of North Carolina Press, 2012) et les colloques triennaux sur les femmes religieuses organisés par le Cushwa Center for the Study of American Catholicism, <http://www.chwr.org $>$.

45. Sarah A. Curtis, Civilizing Habits: Women Missionaries and the Revival of French Empire (New York, Oxford University Press, 2010) et «Traverser les frontières: Philippine Duchesne et les Sœurs du SacréCœur dans le Missouri des années 1820 aux années 1840", Histoire et missions chrétiennes, 17 (2011), p. 59-81. La problématique est élargie spatialement dans la thèse en cours de Gabrielle Guillerm, "The Forgotten French: Catholicism, Colonialism, and Americanness on the Early Trans-Appalachian Frontier", Northwestern University.

46. Philippine Duchesne. Pionnière à la frontière américaine (1769-1852). Euvres complètes rassemblées et éditées par Marie-France Carreel RSCJ et Carolyn Osiek RSCJ (Turnhout, Brepols, 2017). Les écrits des compagnes de Duchesne ne sont édités que pour la période 1818-1823: Philippine Duchesne et ses compagnes, Les années pionnières. 1818-1823. Lettres et journaux des premières missionnaires du Sacré-Cœur aux États-Unis. Textes rassemblés, établis et édités par Chantal Paisant (Paris, Cerf, 2001).

47. Richard J. Janet, In Missouri's Wild. St Mary's of the Barrens and the American Catholic Church, 18181826 (Kirksville, Truman State University Press, 2017). Le chapitre se rapportant à la période concernée ici avait déjà été publié sous le titre "The Era of Boundlessness at St. Mary’s of the Barrens, 1818-1843: A Brief Historical Analysis", Vincentian Heritage Journal 31, 2 (2012), <https:/ via.library.depaul.edu/ $\mathrm{vhj} / \mathrm{vol} 31 /$ iss $2 / 3 />$. Le travail de Janet est exemplaire d'une certaine historiographie catholique (voir son manifeste, "On Catholic History», <https://www.hprweb.com/2014/04/on-catholic-history/>. 
Tableau 2

Les pensionnaires de Sainte-Marie des Barrens, Perryville (Missouri), 1818-1828

\begin{tabular}{|l|l|c|}
\hline \multirow{2}{*}{ Élèves du collège $^{1}$} & Créoles & 9 \\
\cline { 2 - 3 } & Anglo-Américains & 0 \\
\hline \multirow{4}{*}{ Séminaristes missionnaires européens } & Italiens & 9 \\
\cline { 2 - 3 } & Français & 25 \\
\cline { 2 - 3 } & Belges $^{2}$ & 7 \\
\cline { 2 - 3 } & Allemands & 1 \\
\cline { 2 - 3 } & Irlandais & 2 \\
\cline { 2 - 3 } & Espagnols $^{2}$ & 10 \\
\hline \multirow{3}{*}{ Séminaristes nord-américains } & Créoles $^{3}$ & $\mathbf{8 6}$ \\
\cline { 2 - 3 } & Anglo-Américains $^{2}$ & 14 \\
\hline \multicolumn{2}{|l}{ Total } &
\end{tabular}

I. Le registre ne donne que six entrées en 1822 et trois en 1823 , alors que le collège compte une centaine d'élèves en 1830 - avant sa fermeture en I835. II faut supposer que les élèves du collège ont été ensuite comptés sur un registre séparé et disparu.

2. Ils ne sont «belges» que pour faciliter la compréhension:ce sont des ressortissants des Pays-Bas issus du sud catholique du royaume, qui deviendra indépendant en 1830 .

3. Le recrutement est large: le Missouri certes mais aussi Kaskaskia (Illinois), le Haut-Canada, Détroit, la Nouvelle-Orléans.

Source: DeAndreis Rosati Memorial Archives (DePaul University), St Mary's of the Barrens seminary, Box 37, folder I, «Catalogus alumnorum seminarii S. Maria Diocesis Ludovicensis in America Septentrionali».

Il y aurait beaucoup à tirer de ce simple tableau, mais retenons seulement, pour ce qui nous concerne ici, que sur les 77 séminaristes de ces 10 années, un tiers sont des Français (qui constituent près de la moitié des missionnaires européens). Ces 25 jeunes hommes sont issus des réseaux lyonnais et auvergnats et arrivent par vague en fonction des tournées de recrutement des évêques des États-Unis en Europe. Leurs destins sont aussi variés que ceux de n'importe quel migrant. Certains font de très belles carrières, comme Jean-Marie Odin dont $\mathrm{M}^{\mathrm{gr}}$ Rosati disait qu'il était «[s]a consolation et [s]on principal coopérateur [...]. Il est prêt à tout, prêcher, confesser, visiter les malades de jour et de nuit, faire des classes ${ }^{48}$.» Il deviendra évêque de Galveston puis archevêque de La Nouvelle-

48. Archives de l'œuvre de la Propagation de la Foi, fonds de Lyon, F-65 New Orleans, Rosati à Cholleton, 9 juin 1824. 
Orléans ${ }^{49}$. D’autres échouent, prennent conscience que leur place n'est pas au séminaire ou sont exclus : certains qui rentrent en France (Étienne Barreau, Rodolphe Joanoly...) et d'autres dont on aimerait retrouver la trace une fois qu'ils ont quitté Perryville. Et la majorité connaît les affres d'une vie de simple prêtre dans la vallée du Mississippi.

Prenons un exemple: Jean Bouiller, dont le registre ne donne que le nom. Né en 1801 à Roanne dans une famille marchande, il entre au séminaire Saint-Irénée de Lyon en 1821 et y est recruté pour les missions de la Louisiane par $\mathrm{M}^{\mathrm{gr}}$ Dubourg $^{50}$. N'ayant pas achevé sa formation, il est placé à Sainte-Marie des Barrens et devient lazariste. Une fois ordonné et contrairement à ce qu'il espérait sans doute, il n'est pas envoyé dans la région de La Nouvelle-Orléans mais dans les monts Ozarks, en charge des sites miniers de Vieille Mine (Old Mines) et Richwood. Il y mène un travail habituel de prêtre catholique. Il lui faut vivre chez l'habitant faute de mieux, songer à bâtir une église et un presbytère, lutter contre les bals, soutenir ses ouailles aux prises avec le choléra, régler surtout des affaires matrimoniales: des mariages mixtes entre catholiques et protestants et d'autres nécessitant des dispenses de consanguinité dans des familles créoles très endogames. Mais surtout, il doit faire face à une crise, en $1832^{51}$. En conflit violent avec son collègue italien Borgna, il part quelques mois en Louisiane après «deux semaines d'ennuis, de peine et de chagrin", mais est rappelé à Vieille Mine par $\mathrm{M}^{\text {gr }}$ Rosati «sous promesse que Mr B[orgna] n’y mettra plus les pieds " $^{52}$. Il s'agit sans doute d'une incompatibilité d'humeur en raison de l'incapacité de Bouiller d'assurer pleinement son service. En effet, le 21 avril 1832, plus de 80 paroissiens adressaient une pétition à leur évêque demandant le maintien de Borgna pour suppléer Bouiller, car ce dernier était incapable de parler l'anglais. Or $60 \%$ des signataires de la pétition sont anglophones ${ }^{53}$. Le monde a changé : les Créoles sont désormais minoritaires dans la région minière, même parmi la population catholique. D’ailleurs, sur les 39 mariages que Bouiller célèbre entre 1828 et 1834, 17 concernent des couples

49. Patrick Foley, Missionary Bishop: Jean-Marie Odin in Galveston and New Orleans (College Station, Texas A\&M University Press, 2013).

50. Archives du Séminaire Saint-Irénée de Lyon. Une note typographiée tardive intitulée «Départs pour l'Amérique» indique que Jean Bouiller était un vicaire de Roanne né en 1791. Il s'agit d'un homonyme: P1. «Liste des séminaristes entrés au séminaire de Lyon depuis le 1er janvier 1811 » indique le bon Jean Bouiller, né en 1801 à Roanne.

51. Archives de l'Archevêché de Saint-Louis (AASL), Bishop Rosati papers, Box A5, RG 01 B 04.3b, Bouiller à Rosati, 13 août 1828, 10 juillet 1833, 12 mars 1834, 22 août 1834 .

52. AASL, Bishop Rosati papers, Box A5, RG 01 B 04.3b, Bouiller à Rosati, 2 juin 1832, 14 août 1832.

53. AASL, Bishop Rosati papers, Box A5, RG 01 B 04.2, pétition des habitants d'Old Mine et Richwood. 
mixtes, ce qui laisse supposer une lente anglicisation des habitants ${ }^{54}$. Bouiller n'avait plus vraiment sa place dans ce contexte et il finit par partir définitivement en Louisiane. Une nouvelle phase de l'histoire des Français du Missouri s'ouvrait.

\section{|840- | 86 | : LE TEMPS DES MIGRATIONS DE MASSE}

L'admission du Missouri comme État en 1821 est sans conteste une date fondamentale dans l'histoire états-unienne, fruit du compromis de 1820 qui permettait de faire tenir ensemble le Nord et le Sud pour quelques décennies encore $^{55}$. Mais dans l'histoire du peuplement de la région, les changements ne se font pas sentir immédiatement et le tournant date plutôt de la fin des années 1830 et plus encore des années 1840. Le Missouri entre alors, avec le pays entier, dans une nouvelle phase de l'histoire des migrations atlantiques. Celles-ci, pour des raisons européennes autant qu'américaines, s'accélèrent alors largement autour de deux flux majeurs: irlandais et allemand. Le second est bien connu depuis les années 1970: les Allemands du Missouri servirent même en partie à fonder l'étude des communautés migrantes aux États-Unis en mobilisant le concept de chaîne migratoire et les analyses quantitatives $^{56}$. Aujourd'hui, les analyses se font culturelles - quel poids, par exemple, les Allemands, dont beaucoup furent des exilés de la révolution de 1848-1849, eurent-ils dans le débat sur l'esclavage? - mais cette communauté demeure au centre de la réflexion ${ }^{57}$, laissant les Irlandais en arrièreplan, comme d'autres flux, tels ceux venus de France.

Les migrations françaises vers les États-Unis durant ces années ont fait l'objet de quelques travaux. Des historiens ont travaillé les lieux d'arrivée et on connaît les modalités de l'installation à New York, à la NouvelleOrléans, dans la Californie de la ruée vers l'or ou les communautés utopi-

54. Washington County courthouse, Marriage records, book A.

55. William Foley fait de 1821 le terme de son histoire des origines de l'État alors que Stephen Aron en poursuit l'histoire jusqu'aux années 1830. William E. Foley, The Genesis of Missouri: From Wilderness Outpost to Statehood (Columbia, University of Missouri Press, 1989); S. Aron, American confluence...

56. George Helmut Kellner, «The German Element on the Urban Frontier: St. Louis, 1830-1860 ", thèse de doctorat (histoire), University of Missouri-Columbia, 1973; Walter Kamphoefner, The Westfalians : From Germany to Missouri (Princeton, Princeton University Press, 1987); Robert W. Frizzell, Independent Immigrants: A Settlement of Hanoverian Germans in Western Missouri (Columbia, University of Missouri Press, 2007). Il n'y a pas que dans le Missouri que les Allemands ont attiré l'attention, voir par exemple les travaux de Kathleen Neils Conzen, Immigrant Milwaukee: Accomodation and Community in a Frontier City (Cambridge, Harvard University Press, 1976) ou Making Their Own America. Assimilation Theory and the German Peasant Pioneer (New York, Berg, 1990).

57. Luke Ritter, «Sunday Regulation and the Formation of German American Identity in St. Louis, 1840-1860", Missouri Historical Review, 107, 1 (octobre 2012), p. 23-40; Kristen Layne Anderson, Abolitionizing Missouri: German Immigrants and Racial Ideology in Nineteenth-Century America (Baton Rouge, Louisiana State University Press, 2016). 
ques $^{58}$. D’autres ont privilégié les points de départ: l'Alsace et la Lorraine notamment et, dans ce dernier cas, Camille Maire a signalé des départs pour le Missouri et l'existence d'une communauté lorraine à East St. Louis, la banlieue de Saint-Louis sur la rive orientale du Mississippi, dans l'Illinois ${ }^{59}$. Les Français sont, une fois de plus, peu nombreux relativement: en 1860, on compte 5283 natifs de France dans un État de 1182012 habitants, soit $0,4 \%$ de la population. La même année, on compte 88487 personnes nées en Allemagne et 43464 nées en Irlande. Cela dit, là encore, même timides, les flux français informent l'histoire atlantique du Missouri. Les Français qui rejoignent l'État sont comme d'autres Européens le fruit de nouvelles révolutions atlantiques, celles des nations et du capitalisme industriel.

A priori, il serait naturel de se pencher d'abord sur le cas de Saint-Louis, ville en pleine croissance passée de moins de 5000 habitants en 1830 à 160773 habitants en 1860. Curieusement, il y aurait encore beaucoup à dire sur la ville, qui n'a pas reçu de traitement global équivalent à celui, par exemple, de Milwaukee, au temps où les historiens traitaient massivement les données statistiques ${ }^{60}$. Les Français y sont jusqu’à présent passés à peu près inaperçus, alors que les migrations y renouvellent le vieux fonds francophone et laissent planer l'espoir que la ville entretienne sa francité. Louis Cortambert, socialiste utopique qui, au début des années 1830, avait visité l'État en y imaginant tous les possibles, était dans les années 1850 installé à Saint-Louis où il avait épousé une héritière des Chouteau. En 1854, il lançait un journal, la Revue de l'Ouest. Il y annonçait de manière optimiste:

La population française est peu nombreuse encore dans l'Ouest. Nous sommes donc dans l'obligation de prendre au début une allure modeste. Mais la ville de St. Louis étant évidemment destinée à devenir au bout de peu d'années une immense cité, l'un des premiers, sinon le premier centre d'affaires aux États-Unis, il est à croire que la population française y prendra, pour le moins, le même accroissement qu'à New York ${ }^{61}$.

58. M. Polfliet, «Émigration et politisation...»; Carl A. Brasseaux, The Foreign French: Nineteenth Century French Immigration into Louisiana, 1820-1839 (Lafayette, Center for Louisiana Studies, University of Southwestern Louisiana, 1990); Marjorie Bourdelais, La Nouvelle-Orléans: croissance démographique, intégration urbaine et sociale (1803-1860) (Bern, Peter Lang, 2012); A. Foucrier, Le rêve californien...; Michel Cordillot, Utopistes et exilés du Nouveau Monde. Des Français aux États-Unis de 1848 à la Commune (Paris, Vendémiaire, 2013).

59. Nicole Fouché, Émigration alsacienne aux États-Unis (Paris, Publications de la Sorbonne, 1992); Camille Maire, L'émigration des Lorrains en Amérique, 1815-1870 (Metz, Centre de recherche de relations internationales de l’Université de Metz, 1980).

60. L'absence de telles données est particulièrement frappante dans Adam Arenson, The Great Heart of the Republic: St. Louis and the Cultural Civil War (Cambridge, Harvard University Press, 2011).

61. Revue de l'Ouest, 1 (1854), p. 1. Sur Cortambert, voir John Francis McDermott, «Louis Cortambert and the First French Newspapers in St. Louis, 1809-1854 ", The Papers of the Bibliographical Society of America, 
C'était trop s'avancer, que ce soit sur le sort de Saint-Louis - bientôt dépassée par Chicago ${ }^{62}$ - ou sur sa communauté française. Mais il n'en demeure pas moins que la revue de Cortambert, ses articles, ses nombreuses publicités, malgré son unique année d'existence, sont le signe d'une présence qui n'attend que son historien. Je ne ferai pas porter, néanmoins, mon analyse sur ce cas. Mon hypothèse est plutôt que Saint-Louis synthétise la variété des situations françaises à l'échelle de l'État. Un aperçu rapide sur les 419 Français de la $4{ }^{\mathrm{e}}$ circonscription de la ville tendrait à me le confirmer, mais l'hypothèse devra être vérifiée plus avant. Je me propose donc d'observer la présence française à travers cinq échantillons tests: trois villes et deux zones rurales analysées au travers du recensement fédéral de 1860, choisi parce qu'il permet de tirer des leçons de vingt années de changement à la veille du bouleversement majeur que représente la guerre de Sécession.

Saint-Joseph et Hannibal sont les deuxième et troisième plus grosses villes de l'État, mais très loin derrière Saint-Louis, avec 8932 et 6505 habitants. Ce sont deux pôles commerciaux fluviaux, sur le Missouri et le Mississippi, reliés entre eux par une ligne de chemin de fer (celle qui s'avance alors le plus loin vers l'Ouest). Saint-Joseph est également un des points de départ, depuis les années 1840, des grandes routes de l'Ouest, celles des migrants comme celles des marchands. Il s'agit de deux communautés dynamiques, typiques de petites villes du Midwest (mais un Midwest sudiste, à esclaves) où se construisent de fermes hiérarchies sociales en même temps qu'elles sont le théâtre de mouvements incessants de populations ${ }^{63}$. Sainte-Geneviève est un modèle mixte. À bien des égards, elle ressemble, en plus modeste, aux deux cités susdites, notamment parce que, fluviale comme elles, elle attire désormais de forts contingents allemands et anglo-américains et que son économie est diversifiée. Mais elle demeure aussi ce vieux pôle francophone qui lui donne sa singularité culturelle. Le comté de Scott, au sud de Sainte-Geneviève, se distingue par l'existence en son sein d'un township particulier, Moreland, où a été préservée la mémoire de migrations alsa-

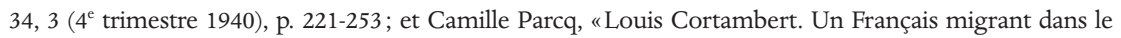
Missouri (1830-1860), mémoire de master (histoire), Université de La Rochelle, 2016.

62. Dans le combat entre les deux villes, c'est historiographiquement aussi Chicago qui a gagné une œuvre classique qui fait défaut à Saint-Louis: William Cronon, Nature's Metropolis: Chicago and the Great West (New York, Norton, 1991).

63. Timothy R. Mahoney, River Towns in the Great West: The Structure of Provincial Urbanization in the American Midwest, 1820-1860 (New York Cambridge University Press, 1990); Provincial Lives : Middle Class Experience in the Antebellum Middle West (New York, Cambridge University Press, 1999) et sa suite, From Hometown to Battlefield in the Civil War Era: Middle Class Life in Midwest America (New York, Cambridge University Press, 2016). Sur Saint-Joseph, voir Robert J. Willoughby, Robidoux's Town: A Nineteenth-Century History of St. Joseph, Missouri (Saint-Joseph, Platte Purchase Publishers, 2006 [1997]). 
ciennes rurales. Le comté de Chariton, lui, exemplifie ce qu'était le Missouri avant la guerre de Sécession: il se situe dans ce que l'on a pris l'habitude de nommer Little Dixie, une vaste zone rurale qui prend en écharpe la vallée du Missouri et où se développe une agriculture esclavagiste de petites propriétés autour de la culture du chanvre ou du tabac. Ce comté comprend donc $27 \%$ d'esclaves au sein de sa population (contre $10 \%$ dans l'ensemble de l'État ${ }^{64}$ ) et les colons qui s'y sont installés proviennent majoritairement du Haut-Sud ${ }^{65}$.

Le premier constat est l'aspect globalement résiduel de la présence française dans l'État hors pôles exceptionnels. Il faut bien sûr tenir compte du biais de ma méthode, car c'est à Saint-Louis qu'il y a le plus de Français. Mais si la grande majorité des Français du Missouri y sont installés, ce n'est pas là qu'ils sont proportionnellement les plus présents. Le deuxième constat est qu'il faut toujours se méfier de certains chiffres: non seulement les recensements fédéraux étatsuniens sont susceptibles d'être imprécis et biaisés $^{66}$ - il faut néanmoins faire avec ces imprécisions et ces biais - mais dans le détail, les données concernant plusieurs natifs de France peuvent poser question. Pour ne prendre qu'un exemple, dans le comté de Chariton, Louis Benecke, 18 ans, est indiqué comme né en France. Mais ses parents et sa sœur de 20 ans sont nés dans le duché de Brunswick et deux autres enfants au foyer, de 3 ans et de 1 an, sont nés au Missouri. Quel peut être, dès lors, le sens de cette naissance française hormis un lieu d'enregistrement légal?

Malgré tout, il faut faire le constat d'une présence diffuse, interstitielle le plus souvent et par moment plus dense. Passons rapidement sur le comté de Chariton. La quasi-absence de Français signale bien la nature de Little Dixie: une manière d'homogénéité culturelle sudiste qui s'est nourrie d'arrivées du Kentucky ou du Tennessee plus que d'immigration. Les Français n'y ont pas leur place, mais ce n'est pas non plus une zone de forte présence allemande. Hannibal, sur le Mississippi, semble dépourvue d'une grande force d'attraction et retient surtout des hommes seuls qui peuvent se marier aux États-Unis. Saint-Joseph est dans une

64. Mais d'une part l'État en comprenait $18 \%$ en 1830 et d'autre part Saint-Louis fait considérablement baisser la moyenne, le comté ne comprenant que $2 \%$ d'esclaves.

65. R. Douglas Hurt, Agriculture and Slavery in Missouri's Little Dixie (Columbia, University of Missouri Press, 1992); Christopher Phillips, Missouri's Confederate: Claiborne Fox Jackson and the Creation of Southern Identity in the Border West (Columbia, University of Missouri Press, 2000); Diane Mutti Burke, On Slavery's Border: Missouri's Small-Slaveholding Households, 1815-1865 (Athens, University of Georgia Press, 2010); Kristen Epps, Slavery on the Periphery: The Kansas-Missouri Border in the Antebellum and Civil War Eras (Athens, University of Georgia Press, 2016).

66. Paul Schor, Compter et classer. Histoire des recensements américains (Paris, éditions de l’EHESS, 2009). 
Tableau 3

Population née en France en 1860

\begin{tabular}{|l|c|c|c|}
\hline & $\begin{array}{c}\text { Population } \\
\text { totale }\end{array}$ & $\begin{array}{c}\text { Natifs } \\
\text { de France }\end{array}$ & $\begin{array}{c}\text { Proportion } \\
\text { de natifs de France }\end{array}$ \\
\hline État du Missouri & 1182012 & 5283 & $0,4 \%$ \\
\hline Ville d'Hannibal & 6505 & 10 & $0,15 \%$ \\
\hline Ville de Saint-Joseph & 8932 & 66 & $0,74 \%$ \\
\hline Ville de Sainte-Geneviève & 1277 & 58 & $4,5 \%$ \\
\hline Comté de Chariton & 12562 & 4 & $0,03 \%$ \\
\hline $4^{e}$ circonscription de Saint-Louis & 15453 & 419 & $2,7 \%$ \\
\hline $\begin{array}{l}\text { Township de Moreland, } \\
\text { comté de Scott }\end{array}$ & 1558 & 132 & $8,5 \%$ \\
\hline
\end{tabular}

Source: recensement fédéral de 1860, roll 609, 613, 632, 644, 657.

Tableau 4

Répartition par type de la population née en France en 1860

\begin{tabular}{|c|c|c|c|c|c|}
\hline & Hannibal & $\begin{array}{l}\text { Saint- } \\
\text { Joseph }\end{array}$ & $\begin{array}{c}\text { Sainte- } \\
\text { Geneviève }\end{array}$ & $\begin{array}{l}\text { Comté de } \\
\text { Chariton }\end{array}$ & $\begin{array}{c}\text { Township } \\
\text { de } \\
\text { Moreland }\end{array}$ \\
\hline Enfants & 1 & 5 & 5 & 1 & 25 \\
\hline $\begin{array}{l}\text { Enfants exerçant une } \\
\text { profession }\end{array}$ & & & 7 & & 14 \\
\hline $\begin{array}{l}\text { Couples dont les deux } \\
\text { membres sont nés en France }\end{array}$ & & 15 & 14 & 1 & 27 \\
\hline $\begin{array}{l}\text { Couples dont l'épouse est } \\
\text { née en France }\end{array}$ & 1 & 7 & 2 & & 15 \\
\hline $\begin{array}{l}\text { Couples dont le mari est } \\
\text { né en France }\end{array}$ & 4 & 7 & 8 & 1 & 12 \\
\hline Célibataires femmes & & & 3 & & 5 \\
\hline Célibataires hommes & 3 & 13 & 3 & & \\
\hline Veuves et veufs & & 4 & 2 & & 6 \\
\hline Enfant isolé & 1 & & & & 1 \\
\hline
\end{tabular}

Source: recensement fédéral de 1860, roll 609, 613, 632, 644, 657 . 


\section{Carte 2}

\section{Les natifs de France dans le Missouri de I860}

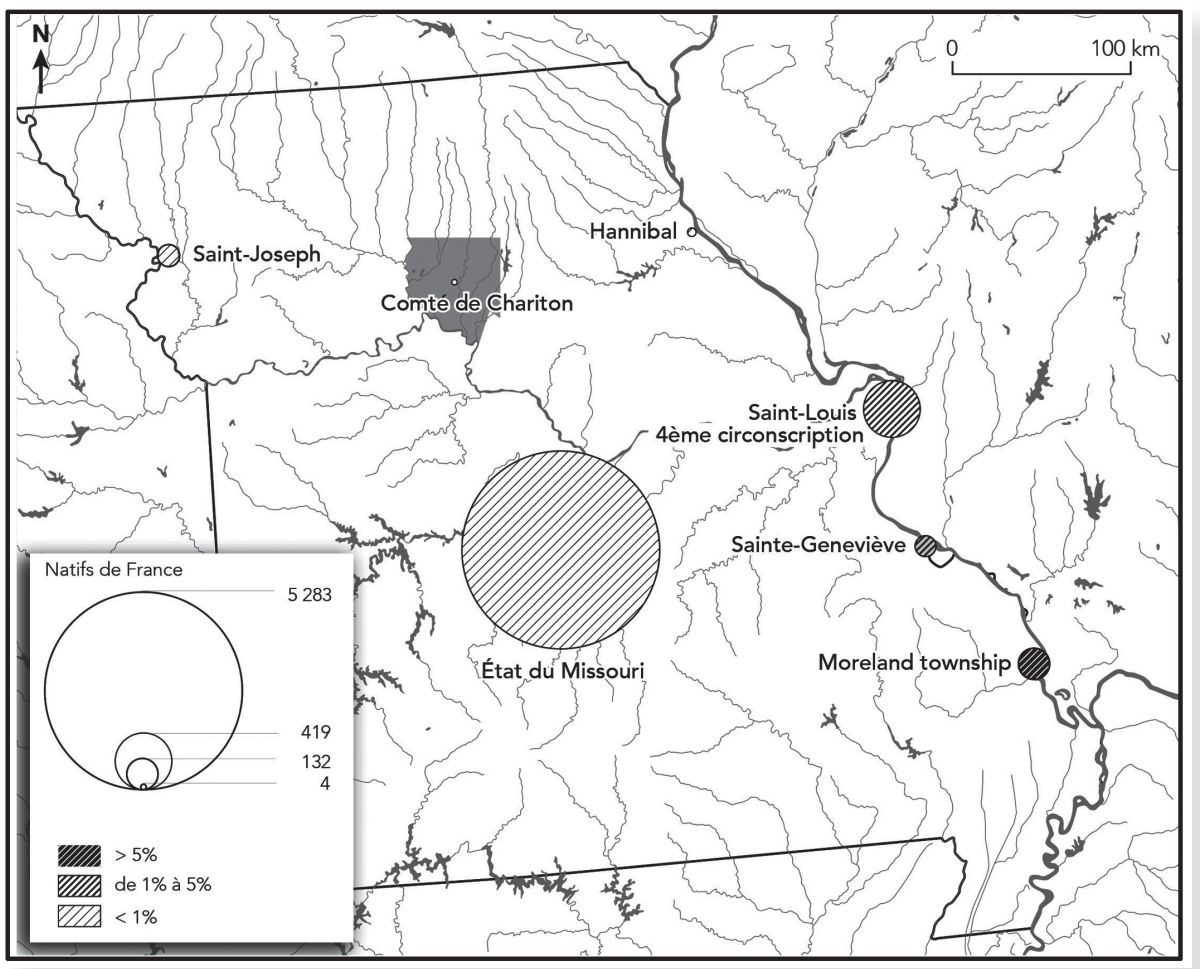

Conception Soazig Villerbu/Réalisation Pascal Brunello, CTIG, Université de La Rochelle

situation différente. Son caractère de porte de l'Ouest ouverte sur la Prairie et la vallée du Missouri, son fort dynamisme commercial, en font un pôle capable de retenir l'attention de migrants - dont au demeurant il faudrait mesurer également la durée d'installation - aux profils variés mais correspondant bien à une ville de ce type. On y compte beaucoup de célibataires, hommes en quête d'opportunités qu'est censée offrir la ville, mais aussi des couples de Français travaillant dans le commerce (les saloons notamment) et l'artisanat, et autant de couples mixtes. SaintJoseph est ainsi une sorte de petit Saint-Louis.

Sainte-Geneviève et le township de Moreland se distinguent par la proportion de natifs de France en leur sein, mais avec deux profils différents révélés à la fois par la mémoire locale et généalogique, les anthroponymes (avec leurs limites) et la nature des mariages mixtes. À Sainte- 
Geneviève, les «Français», pourtant peu nombreux en valeur absolue, forment une véritable marqueterie. On y trouve de manière condensée la plupart des manières de s'installer dans le Missouri, en couple ou non, riche ou pauvre, dans les métiers du commerce, de l'artisanat ou de la culture (au sens large, du prêtre au professeur de musique). Moreland est beaucoup plus homogène. C'est un monde de fermiers assez aisés venus d'un vaste espace germanique où les Alsaciens sont bien représentés ${ }^{67}$. Il y a autant de couples mixtes que de couples "français» et les femmes n'épousent alors que des hommes issus des pays allemands, tandis que les hommes bénéficient d'un choix plus large qui peut les faire sortir de leur communauté linguistique. La situation est d'ailleurs similaire dans les autres cas. Dans les 5 lieux choisis, 33 mariages mixtes sur 54 impliquent un conjoint allemand, mais le contraste entre les genres est frappant: 21 femmes sur 24 épousent un Allemand alors qu'il ne s'agit que de 11 hommes sur 30, comme si la clôture communautaire ne s'imposait qu'aux femmes. Car le mariage avec un Allemand, s'il peut être considéré comme un mariage mixte puisque contracté entre deux personnes de nationalité différente, est aussi dans le contexte missourien, et de manière extrême dans le township de Moreland, une façon au contraire de ne pas sortir de son monde, de s'expatrier pour mieux reproduire des solidarités européennes.

Alsaciens et Alsaciennes - fort peu, voire pas du tout francophones contribuent en fait à la création, avec leurs voisins badois ou prussiens, de la communauté (et de la nation) allemande. Ils rejouent dans la vallée du Mississippi (et celle du Missouri de manière moindre) un espace rhénan qu'il faudrait penser globalement pour les populations ici généralement catholiques comme Anny Bloch-Raymond l'a fait pour les populations juives de Louisiane $^{68}$. Il est en effet frappant de constater que ces Allemands sont issus du grand-duché de Bade, des royaumes de Wurtemberg et de Bavière (avec son annexe du Palatinat), et de la Prusse qui inclut alors la Rhénanie. Avec l'Alsace, il s'agit d'un seul grand ensemble rhénan qui s'expatrie et la notion même de mariage mixte s'efface. Le phénomène gagnerait alors à une comparaison systématique avec une migration qui a davantage attiré les chercheurs français, celle des Basques (et des Béarnais) marquée de même

67. Ce monde correspond à celui décrit par N. Fouché, Émigration alsacienne...

68. Anny Bloch-Raymond, Des berges du Rhin aux rives du Mississippi. Histoire et récits des migrants juifs (Paris, Michel Houdiard, 2009). 
Tableau 5 Pays de naissance des épouses et maris dans les cas
de couples mixtes

\begin{tabular}{|l|l|c|c|}
\hline \multirow{4}{*}{ Pays allemands } & \multicolumn{1}{|c|}{$\begin{array}{c}\text { Pays de naissance } \\
\text { du mari lorsque l'épouse } \\
\text { est native de France }\end{array}$} & $\begin{array}{c}\text { Pays de naissance } \\
\text { de l'épouse quand le mari } \\
\text { est natif de France }\end{array}$ \\
\hline & Bavière & 8 & 2 \\
\cline { 2 - 4 } & Prusse & 2 & 0 \\
\cline { 2 - 4 } & Bade & 5 & 6 \\
\cline { 2 - 4 } & Würtemberg & 0 & 1 \\
\cline { 2 - 4 } & "Allemagne» & 6 & 2 \\
\hline Total pays allemands & & $\mathbf{2 1}$ & $\mathbf{1 1}$ \\
\hline \multirow{3}{*}{ États américains } & Missouri & 0 & 5 \\
\cline { 2 - 4 } & États du Nord & 1 & 5 \\
\cline { 2 - 4 } & États du Sud & 0 & $\mathbf{1 4}$ \\
\hline \multirow{2}{*}{$\begin{array}{l}\text { Total } \\
\text { États américains }\end{array}$} & & $\mathbf{1}$ & $\mathbf{5}$ \\
\hline Autres & & $\mathbf{2}$ & 4 \\
\hline
\end{tabular}

Source: recensement fédéral de 1860, roll 609, 613, 632, 644, 657.

par une frontière d’État dans la région de départ qui peut s'étioler dans le déplacement ${ }^{69}$.

Ainsi, en 1860, les Français du Missouri se caractérisent-ils surtout par la variété de leurs itinéraires. Dans le township de Moreland, il s'agit par exemple de Teresa Messmer, 41 ans, épouse de Charles Messmer, un fermier issu du pays de Bade dont la terre est évaluée à $400 \$$ et les biens personnels à $200 \$$. Cette modestie s'explique sans doute par l'arrivée récente du couple dans le comté de Scott: leurs sept premiers enfants, entre 5 et 17 ans, sont nés dans l'Ohio et seule leur dernière, Rozalia, 3 ans, a vu le jour dans le Missouri. Leur migration date donc du début des années 1840 et ils ont suivi le chemin de l'Ouest. Qu'ont-ils de commun avec Jean et Clémence Gérard? Âgés de 48 et de 49 ans, ceux-ci habitent à Sainte-Geneviève avec trois enfants: Gustave, 19 ans, est né en France, mais Ernest et Marie, 9 et 2 ans,

69. Henry de Charnisay, L'émigration basco-béarnaise en Amérique (Biarritz, J \& D éditions, 1996 [1947]); Adrian Blazquez, dir., L'émigration basco-béarnaise aux Amériques au XIXé siècle (Orthez, Éditions Gascogne, 2005); Laurent Dornel, dir., Des Pyrénées à la Pampa. Une histoire des migrations d'élites (XIXe-XXe siècles) (Pau, Presses de l'Université de Pau et des Pays de l’Adour, 2013); Marie-Pierre Arrizabalaga, «Immigration, intégration, genre. Une communauté française en Californie, 1880-1940», mémoire inédit d’HDR (histoire), Université Paris 1-Panthéon-Sorbonne, 2015. 
sont missouriens de naissance. Jean est charpentier et a réussi sa migration, doté qu'il est de 1800 \$ de biens immobiliers. Sans doute les époux ont-ils trouvé à Sainte-Geneviève la ville qui leur convenait, dans sa spécificité. Leur vie diffère de celle des époux Charles et Joséphine Nigard. Il est charpentier également, leur fils de 4 ans est né à New York, tandis que leur fille de 3 ans est native du Missouri. Installés dans un village du comté de Chariton très peu de temps après leur débarquement sur la côte Est, ils y sont bien seuls et leur assimilation rapide ne fait guère de doute s'ils décident de rester sur place. À Saint-Joseph, les époux Hartley, venus du Vermont, tiennent un hôtel. Parmi les membres du personnel, Jean C. Barre: né en France, il a 21 ans, est célibataire et tient le bar de l'hôtel. À Hannibal, Jacob Fity, charpentier né en France et âgé de 24 ans, loge chez S. R. Millars, un cordonnier aisé qui héberge également cinq hommes (quatre AngloAméricains et un Allemand) travaillant au chemin de fer. Ces cas piochés au hasard du recensement dressent une sorte de portrait kaléidoscopique d'un ensemble aux contours flous, ces «Français» du Missouri au début d'un âge des migrations de masse dans lequel ils perdent leurs spécificités, alors que jusque-là leurs migrations s'inscrivaient souvent dans des contextes français ou missourien très particuliers.

\section{CONCLUSION}

Benjamin Tarpin et Ernest Tasset sont deux Français de Saint-Louis lorsque la guerre de Sécession éclate. Le premier s'engage dans les troupes de l'Union dès octobre 1861 et n'est démobilisé qu'au retour de la paix, après avoir participé à la campagne victorieuse de Grant ponctuée par les batailles de Iuka, Corinth, Champion Hill et le siège de Vicksburg. Le deuxième n'est enrôlé qu'en août 1864 et a juste le temps de contrer avec son unité la terrible, parce que désespérée, campagne du Tennessee menée par le général sudiste John Bell Hood. Après la guerre, tous deux rentrent en France en bénéficiant des services consulaires - une sorte de rapatriement sanitaire. C’est la mère de Tarpin qui avait manifesté son désir de retour dès 1864 , mais en exigeant de pouvoir attendre que son fils rentre du front pour le rapatrier avec elle. C'est Tasset lui-même, par contre, qui en appelle au vice-consul, car une fois revenu de la guerre, une maladie des yeux l'empêche de retrouver un emploi ${ }^{70}$.

70. Les itinéraires des deux soldats sont reconstitués grâce à la base de données en ligne des soldats du Missouri, <https://s1.sos.mo.gov/records/archives/archivesdb/soldiers/>. Pour le reste, Centre des archives diplomatiques de Nantes. New York, 474PO/1/109: Relations New York-autres postes. Chemise «New York. Postes français aux E.U. Saint Louis 1841-1904», Levasseur (vice-consul à Saint-Louis) au consul général de New York, 11 février 1865, 23 février 1865, 23 septembre 1865, 7 octobre 1865. 
La situation est intéressante à au moins deux égards. D’abord parce qu'elle soulève ce qui signifie être français dans le Missouri: ces deux hommes se sont engagés pour le Nord, donc pour défendre l'Union, les États-Unis, ce pays où ils s'étaient installés, dans une logique de défense nationale. Et pourtant une fois la victoire acquise, ils «redeviennent» français en utilisant les services consulaires pour rentrer au pays. Les choix sont donc complexes et multifactoriels; on peut toujours trouver d'infinies nuances à la logique de nationalisation des Français par la guerre décrite par Farid Ameur ${ }^{71}$. Ensuite parce Tarpin et Tasset, somme toute, ont beaucoup en commun avec les Guibourd, les Rozier, les Bouiller, les Gérard ou les Munsch qui les ont précédés depuis la fin du XVIII siècle. Les historiens ont en effet reconceptualisé récemment la guerre de Sécession dans son cadre atlantique, en y voyant un conflit national au cœur d'une grande séquence de conflits de ce type sur les deux rives de l'océan ${ }^{72}$. De ce fait, les itinéraires de Tarpin et Tasset dans le Missouri (et retour) sont aussi atlantiques que le furent ceux de leurs prédécesseurs. Et la confluence continentale que décrivait Stephen Aron ${ }^{73}$ peut donc aussi bien être un point d'ancrage d'une histoire atlantique pensée à différentes échelles en observant les Émigrés administrant la Haute Louisiane espagnole, les jeunes séminaristes de la Restauration lancés dans la conquête des âmes dans le Missouri étatsunien, les marchands tentant de réinventer des circuits commerciaux dans un univers capitaliste en évolution, les Alsaciens jouant des frontières et des identités nationales dans les années 1850, ou ces jeunes engagés de la guerre de Sécession qui pensent deux nations à la fois.

71. Farid Ameur, Les Français dans la guerre de Sécession, 1861-1865 (Rennes, Presses Universitaires de Rennes, 2016).

72. Thomas Bender, Nation among Nations: America's Place in World History (New York, Hill and Wang, 2006); Douglas R. Egerton, «Rethinking Atlantic Historiography in a Postcolonial Era: The Civil War in Global Perspective", Journal of the Civil War Era, 1, 1 (mars 2011), p. 79-95; Paul Quigley, Shifting Grounds: Nationalism and the American South, 1848-1865 (New York, Oxford University Press, 2012); Andre M. Fleche, The Revolution of 1861: The American Civil War in the Age of Nationalist Conflict (Chapel Hill, University of North Carolina Press, 2012); David T. Gleeson et Simon Lewis, dir., The Civil War as Global Conflict: Transnational Meanings of the American Civil War (Columbia, University of South Carolina Press, 2014); Don H. Doyle, The Cause of All Nations: An International History of the American Civil War (New York, Basic Books, 2014).

73. S. Aron, American confluence... 